\title{
Pectobacterium colocasium sp. nov. isolated from taro (Colocasia esculenta)
}

\author{
1.1 Author names
}

Diksha Klair, Dario Arizala, Shefali Dobhal, Gamze Boluk, Anne M. Alvarez and

Mohammad Arif*

\subsection{Affiliation}

Department of Plant and Environmental Protection Sciences, University of Hawaii at Manoa,

Honolulu, HI 96822

1.3 Corresponding author

arif@hawaii.edu; Phone: +1-808-956-7765

\subsection{Keyword}

Pectobacterium; soft rot; taro; taxonomy; MLSA; 16S rRNA; Biolog

\author{
1.5 Repositories:
}

CP091064; MZ542535 - MZ542540; OM457660 
Pectobacterium, agenus comprising gram-negative, pectinolytic phytopathogens, is responsible for economic losses in a wide host range of plants. In this study, the bacterial strains PL152 ${ }^{\mathrm{T}}$ and PL155 were isolated from taro corms in Hawai'i in 2018, and characterized using genomic and biochemical assays. The Next Generation Sequencing technologies, Oxford Nanopore MinION and Illumina NovaSeq, were used for whole genome sequencing of the PL152 $2^{\mathrm{T}}$ strain. Short and long reads were assembled using the Unicycler tool accessible at the bioinformatic resource center, and PATRIC (PathoSystems Resource Integration Center) was used to generate a more accurate and reliable "hybrid" assembly. The $16 \mathrm{~S}$ rRNA analysis of PL $152^{\mathrm{T}}$ with type strains of other known Pectobacterium species showed a close relationship with $P$. fontis. Multi-locus sequence analysis using nine housekeeping genes (dnaA, gapA, gyrB, recA, dnaN, rpoS, mdh, rpoA and dnaK) differentiated strain PL152 ${ }^{\mathrm{T}}$ from other species of Pectobacterium and formed a unique and well-defined clade. The concurrent results of average nucleotide identity (ANI) and digital DNA-DNA hybridization, with calculated values lower than 95 and 70\%, respectively, supported the delineation of a novel bacterial species. Here, we proposed Pectobacterium colocasium, strain PL152 ${ }^{\mathrm{T}}\left(=\mathrm{ICMP} 24362^{\mathrm{T}}\right.$; LMG $32536^{\mathrm{T}}$ ) and PL155 as a novel species in the genus Pectobacterium.

\section{INTRODUCTION}

The genus Pectobacterium (formerly Erwinia) belonging to the family Proteobacteriaceae, is a gram-negative, fermentative, rod-shaped phytopathogen, with an ability to macerate plant cell walls by secreting multiple plant-cell wall degrading enzymes (PCWDEs)[[1, 2].

Pectobacterium ranked among the top ten scientifically and economically studied plant pathogenic bacteria due to its wide host range and the economic losses associated with soft rot diseases [3]. Species within Pectobacterium cause a broad spectrum of symptoms, 
including soft rot, blackleg, and wilt on many crops [4]. In addition to the broad host range, species within this genus have also been isolated from soil, groundwater, and invertebrates [5].

The taxonomy of the genus Pectobacterium has been subjected to constant remodeling, reevaluation, and reorganization, with the simultaneous development of new bacterial classification techniques (Figure 1). For instance, next generation sequencing (NGS), which provides the whole genome sequence, enables analysis of taxonomically complex genera and comparisons of phylogenetic/evolutionary relationships of different species and subspecies of plant pathogenic genera [6, 7]. Currently, Pectobacterium genus includes 20 recognized or proposed species till date. The recognized species includes Pectobacterium actinidiae $[8,9]$, $P$. versatile [8, 10], P. brasiliense [8, 11, 12], P. odoriferum [8, 13], P. aquaticum [14], $P$. aroidearum [15], P. atrosepticum [13, 16], P. betavasculorum $[13,16]$, P. wasabiae $[13,16]$, P. fontis [17], P. punjabense [18], P. cacticida [13, 19, 20], P. carotovorum [13, 16, 21], P. parmentieri [22], P. parvum [23], P. polaris [24], P. polonicum [25], and P. quasiaquaticum [26]. However, two additional species $P$. peruviense and $P$. zantedeschiae $[27,28]$, yet to be validated by the ad hoc committee [29]. Presently, not even the draft genome is avaliable for P. cacticida in any databases. In this current study, two strains, $\mathrm{PL}_{152^{\mathrm{T}}}$ (ICMP $24362^{\mathrm{T}}$; LMG $32536^{\mathrm{T}}$ ) and PL155, were analyzed and proposed as a novel species, 'Pectobacterium colocasium'.

\section{ISOLATION AND BIONOMICS}

The strains PL152 ${ }^{\mathrm{T}}$ and PL155, referred to hereafter as $P$. colocasium, were isolated from soft rot infected taro corm samples collected in Hawaii in 2018. Infected taro corms were surface sterilized with $0.6 \%$ sodium hypochlorite solution for 30 s, followed by three consecutive rinses for 30s each in distilled water. The corm tissues were aseptically macerated in a $1.5 \mathrm{ml}$ 
Eppendorf tube with a sterile pestle, streaked onto Crystal Violet Pectate (CVP) medium [30] for isolation of the colonies within characteristic cavities caused by pectin degradation. Colonies that produced pits were purified by re-streaking the bacteria onto nutrient agar supplemented with $0.4 \%$ dextrose during two consecutive rounds, and confirmed with a PCR assay using Pectobacterium specific primers [31]. The purified cultures obtained from a single colony were stored at $-80^{\circ} \mathrm{C}$ in $25 \%$ glycerol (25:75, Glycerol:LB liquid medium).

\section{MULTI-LOCUS SEQUENCE ANALYSIS}

The bacterial DNA was isolated from purified colonies using DNeasy Blood and Tissue kit following the manufacturer's protocol (Qiagen, Valencia, CA). For identification and initial taxonomic classification of novel bacterial strains PL152 ${ }^{\mathrm{T}}$ and PL155, the isolated DNA was amplified using three housekeeping genes - chromosomal replication initiator protein DnaA (dnaA), glyceraldehyde-3-phosphate dehydrogenase (gapA), and DNA topoisomerase ATPhydrolyzing subunit B $($ gyrB $)$ primers $[32,33]$. The amplified PCR product was enzymatically cleaned using ExoSAP-IT (Affymetrix, Santa Clara, CA) sequenced at Genewiz facility (La Jolla, CA) using both forward and reverse primers, and further aligned and manually edited to remove errors using Geneious R10 [34]. The consensus sequences of dnaA, gapA and gyrB gene sequences for PL152 ${ }^{\mathrm{T}}$ and PL155 strains were submitted to the NCBI GenBank database under the accession numbers MZ542535 - MZ542540. Taxonomic classification and analyses were performed using 51 different bacterial strains, including novel strains of P. colocasium (PL152 ${ }^{\mathrm{T}}$ and PL155), 48 other species of Pectobacterium and D. zeae $\mathrm{ECl}^{\mathrm{T}}$ as an outgroup. The sequences of all Pectobacterium and Dickeya strains used in the analysis were retrieved from the NCBI GenBank genome database in July 2020, with an exception of newly added P. quasiaquaticum (retrieved in January 2022) (Supplemental Table 1). The sequences were aligned with ClustalW, trimmed and concatenated using 
Geneious R10 [34]. MEGA11 software [35] was used to generate the phylogenetic tree using the Maximum Likelihood method with a bootstrap test of 1,000 replicates (Supplemental Figure 1). Based on phylogenetic tree analysis, the novel strains (PL152 ${ }^{\mathrm{T}}$ and PL155) clustered together, indicating clonality, and formed a novel clade which was well differentiated from other Pectobacterium species. To refine the taxonomic classification and delineation of a novel species, Multi-Locus Sequence Analysis (MLSA) [36]was performed with nine housekeeping genes - dnaA, gapA, gyrB, recominase RecA (recA), DNA polymerase III subunit beta (dnaN), RNA polymerase sigma factor RpoS (rpoS), malate dehydrogenase $(m d h)$, DNA-directed RNA polymerase subunit alpha $(r p o A)$, and molecular chaperone DnaK (dnaK) using 54 bacterial species including novel strain PL152 ${ }^{\mathrm{T}}$ and other Pectobacterium and Dickeya species retrieved from NCBI GenBank database (Supplemental Table 1). The sequences were aligned using ClustalW, trimmed and concatenated using Geneious R10 [34]. Phylogenetic analysis was performed with MEGA11 software using the Maximum Likelihood method and Tamura- Nei model with a 1,000 bootstrap replicates (Figure 2). Based on MLSA analysis, a novel strain of $P$. colocasium branched at the roots of other Pectobacterium species, forming a distinctively separate and a novel branch delineating it as a novel Pectobacterium species. Pectobacterium colocasium rooted close to $P$. aroidearum CFBP8737, KC20 and L6, shown as its closest neighbor species.

\section{S rRNA GENE ANALYSIS}

To confirm and validate the generic level identification of novel bacterial strain PL152 ${ }^{\mathrm{T}}, 16 \mathrm{~S}$ rRNA analysis was performed with all 19 type strains of Pectobacterium species, and D. zeae $\mathrm{EC}^{\mathrm{T}}$, as an outgroup, avaiable in NCBI database. The 16s rRNA sequences of $P$. actinidiae $\mathrm{KKH}^{\mathrm{T}}$, . carotovorum $\mathrm{NCPPB} 12^{\mathrm{T}}$, P. aroidearum $\mathrm{SCRI} 109^{\mathrm{T}}$, P. brasiliense LMG $21371^{\mathrm{T}}$, P. polaris $\mathrm{NIBIO} 1006^{\mathrm{T}}, P$. parvum $\mathrm{s} 0421^{\mathrm{T}}, P$. odoriferum $\mathrm{LMG}^{2} 7566^{\mathrm{T}}, P$. versatile 
CFBP6051 ${ }^{\mathrm{T}}, P$. aquaticum A212-S19-A16 ${ }^{\mathrm{T}}, P$. zantedeschiae $9 \mathrm{M}^{\mathrm{T}}, P$. fontis $\mathrm{M} 022^{\mathrm{T}}, P$. polonicum DPMP $315^{\mathrm{T}}$, P. punjabense $\mathrm{SS} 95^{\mathrm{T}}$, P. parmentieri RNS08-42-1A $\mathrm{A}^{\mathrm{T}}$, P. wasabiae CFBP $3304^{\mathrm{T}}$, P. atrosepticum $\mathrm{LMG}^{2} 386^{\mathrm{T}}$, P. peruviense IFB5232 ${ }^{\mathrm{T}}$, P. betavasculorum $\mathrm{CFBP} 2122^{\mathrm{T}}$, P. quasiaquaticum A477-S1-J17 ${ }^{\mathrm{T}}$, and D. zeae $\mathrm{EC}^{\mathrm{T}}$ were retrieved from NCBI GenBank database (Supplemental Table 2). The 16S rRNA gene sequence of PL152 ${ }^{\mathrm{T}}$ was extracted from the whole genome sequence of PL152 ${ }^{\mathrm{T}}$ and was submitted to NCBI GenBank with accession number OM457660. Based on 16S rRNA analysis, PL152 ${ }^{\mathrm{T}}$ clustered with other Pectobacterium species, confirming its identity within Pectobacterium genus (Figure 3). The PL152 ${ }^{\mathrm{T}}$ was closely rooted to $P$. fontis $\mathrm{M} 002^{\mathrm{T}}$ strain in this analysis.

\section{GENOME CHARACTERIZATION}

Bacteria from stored pure culture of PL152 ${ }^{\mathrm{T}}$ were cultured on nutrient agar supplemented with $0.4 \%$ dextrose for total genomic DNA isolation. A half loopful of pure bacterial culture was scrapped for DNA isolation using QIAGEN Genomic-tip 100/G (Qiagen, Valencia, CA) and quantified using the Qubit dsDNA HS kit and Qubit 4 (Thermo Fisher Scientific, Waltham, MA), and analyzed on $1.5 \%$ agarose gel. The library was prepared using Rapid Barcoding kit (SQB-RBK004) (Oxford Nanopore Technologies, Oxford Science Park, UK) with $400 \mathrm{ng}$ of high-quality genomic DNA, and sequencing was performed using a MinION vR9.4 flow-cell following the manufacturer instructions (Oxford Nanopore Technologies, Oxford, UK). Sequencing data generated in the flow-cell was monitored in real time using the MinKNOW software (version 4.0.20). The obtained FAST5 sequences from MinION were base-called using MinKNOW (version 4.0.20). Later, the obtained long reads were corrected using the tool "Correct Long Reads (beta)" of the CLC Genomics Workbench version 20.0. (Qiagen), with default parameters. Additionally, Illumina DNA library was prepared using Seqwell plexWell LP384 Library Preparation kit (seqWell, Beverly, MA) using 10 ng gDNA. 
The prepared library was amplified with 8 PCR cycles, analyzed using Bioanalyzer 2100 (Agilent, Santa Clara, CA), quantified with Qubit, and combined into two pools at equimolar ratios. The library pool was quantified by qPCR with a Kapa Library-Quant kit (Kapa Biosystems/Roche, Basel, Switzerland) and sequenced on an Illumina NovaSeq system (Illumina, San Diego, CA) with paired-end 150-bp reads. The Illumina library preparation and sequencing was performed at Novogene facility (Sacramento, CA). The Illumina pairedend short reads along with the corrected long reads from Oxford Nanopore were used to create an accurate and complete hybrid assembly using the Unicycler assembly pipeline version 0.4.8 [37]with default parameters, as plugged in the web-server PathoSystems Resource Integration Center (PATRIC) [38]. The generated complete genome sequence was annotated using the Prokaryotic Genome Annotation Pipeline (PGAP) version 4.10 and Rapid Annotation using Subsystem Technology (RAST) toolkit [39]. The genome of PL152 ${ }^{\mathrm{T}}$ has a total of 5,019,495 bp and DNA G+C content of $54.6 \mathrm{~mol} \%$. The assembled genome was submitted to the NCBI GenBank genome database under the accession number CP091064.

To assign the $P$. colocasium strain $\mathrm{PL} 152^{\mathrm{T}}$ as a novel species, the average nucleotide identity (ANI) and digital DNA-DNA hybridization (dDDH) was calculated using Qiagen CLC Genomics workbench and Type (Strain) Genome Server (https://tygs.dsmz.de/), respectively. However, the ANI value for $P$. peruviense A97-S13-F16 strain was calculated against $P$. quasiaquaticum A477-S1-J17 ${ }^{\mathrm{T}}$ and P. parvum Y1 using EzBiocloud ANI calculator [40], and dDDH for $P$. quasiaquaticum against all other bacterial strains including in the analysis was calculated using Genome-to-Genome Distance Calculator 3.0 (GGDC) [41] . ANI, defining the nucleotide-level genomic similarity of PL152 ${ }^{\mathrm{T}}$ strain genome against other Pectobacterium species genomes and D. zeae strain EC1, was below the suggested cut-off value of $95-96 \%$ to delineate bacterial species [42] . The ANIm values for P. colocasium strain PL152 ${ }^{\mathrm{T}}$ for analysed Pectobacterium species ranged between $88.26-91.46 \%$ : $P$. 
aquaticum A212-S19-A $16^{\mathrm{T}}$ (90.42\%), P. polonicum DPMP $315^{\mathrm{T}}$ (88.92\%), P. actinidae $\mathrm{KKH}^{\mathrm{T}}(89.95 \%)$, P. fontis $\mathrm{M} 022^{\mathrm{T}}(88.26 \%)$, P. betavasculorum $\mathrm{NCPPB}^{2795^{\mathrm{T}}}(88.85 \%), P$. polaris $\mathrm{NIBIO} 1006^{\mathrm{T}}(90.76 \%)$, P. brasiliense $\mathrm{LMG}^{21371^{\mathrm{T}}}(91.02 \%)$, P. carotovorum NCPPB $312^{\mathrm{T}}(90.65 \%), P$. aroidearum L6 $(93.87 \%), P$. versatile CFBP6051 ${ }^{\mathrm{T}}(90.44 \%), P$. wasabiae CFBP3304 ${ }^{\mathrm{T}}(88.52 \%)$, P. odoriferum BC S7 (90.24\%), P. parmentieri RNS08-42$1 \mathrm{~A}^{\mathrm{T}}(88.44 \%)$, P. punjabense SS95 ${ }^{\mathrm{T}}(88.78 \%)$, P. parvum Y1 $(91.46 \%)$, P. zantedeschiae PC2 (88.55\%), P. atrosepticum NCPPB549 ${ }^{\mathrm{T}}$ (88.9\%) P. peruviense A97-S13-F16 (88.28\%), and P. quasiaquaticum A477-S1-J17 ${ }^{\mathrm{T}}$ (91.12\%) (Table 1). The predicted dDDH values for PL152 $2^{\mathrm{T}}$ as a query against other strains of Pectobacterium species ranged between 21.244.7\%; A212-S19-A $16^{\mathrm{T}}(41.3 \%), \mathrm{DPMP} 15^{\mathrm{T}}(36.4 \%), \mathrm{KKH}^{\mathrm{T}}(39.2 \%), \mathrm{M}^{2} 2^{\mathrm{T}}(34.8 \%)$,

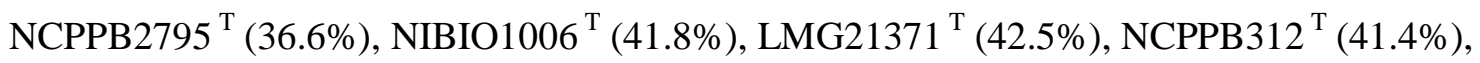

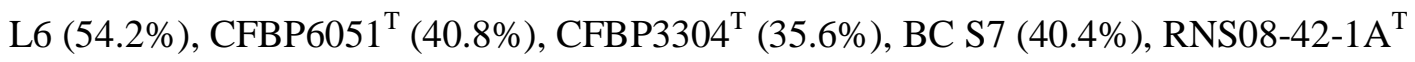
(35.4\%), SS95 ${ }^{\mathrm{T}}(36 \%), \mathrm{Y} 1$ (44.7\%), PC2 (35.3\%), NCPPB549 ${ }^{\mathrm{T}}$ (36.4\%), A97-S13-F16 (34.6\%), and $\mathrm{A} 477-\mathrm{S} 1-\mathrm{J} 17^{\mathrm{T}}(43.1 \%)$ and D. zeae $\mathrm{EC}^{\mathrm{T}}(21.2 \%)$ were lower than the threshold value of 70\% (Wayne et al. 1987) (Table 1). Therefore, based on the concurrence results of comparative genomic, whole genome analysis and clustering of strains in MLSA analysis, authors clearly propose Pectobacterium colocasium sp. nov. PL152 ${ }^{\mathrm{T}}$ and PL155 strains as a novel Pectobacterium species. 
Table 1. Average nucleotide identity (ANI) and digital DNA-DNA hybridization (dDDH; in bold type) estimations of Pectobacterium colocasium strain PL152 ${ }^{\mathrm{T}}$ (=ICMP $24362^{\mathrm{T}}$; LMG $32536^{\mathrm{T}}$ ) against other species of Pectobacterium genus and Dickeya zeae EC1 ${ }^{\mathrm{T}}$ (an outgroup). The upper right triangle and lower left triangle represent ANIm and dDDH values, respectively.

\begin{tabular}{|c|c|c|c|c|c|c|c|c|c|c|c|c|c|c|c|c|c|c|c|c|c|c|}
\hline \multicolumn{23}{|c|}{ ANI value } \\
\hline \multirow{2}{*}{\multicolumn{2}{|c|}{ Species }} & 1 & 2 & 3 & 4 & 5 & 6 & 7 & 8 & 9 & 10 & 11 & 12 & 13 & 14 & 15 & 16 & 17 & 18 & 19 & 20 & 21 \\
\hline & & \multirow[t]{2}{*}{ 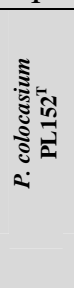 } & \multirow{3}{*}{ 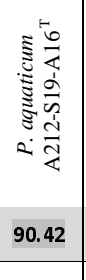 } & \multirow{2}{*}{ 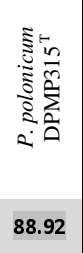 } & \multirow{2}{*}{ 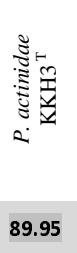 } & \multirow{2}{*}{ 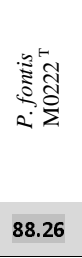 } & \multirow{2}{*}{ 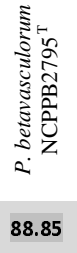 } & \multirow{2}{*}{ 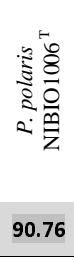 } & \multirow{2}{*}{ 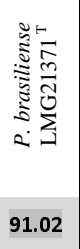 } & \multirow{2}{*}{ 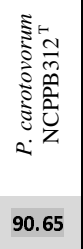 } & \multirow{2}{*}{ 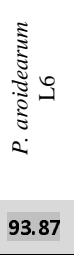 } & \multirow{2}{*}{ 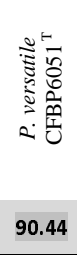 } & \multirow{2}{*}{ 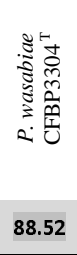 } & \multirow{2}{*}{ 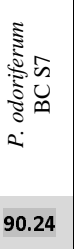 } & \multirow{2}{*}{ 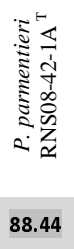 } & \multirow{2}{*}{ 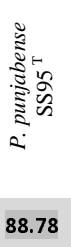 } & \multirow{2}{*}{ 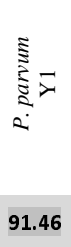 } & \multirow{2}{*}{ 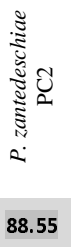 } & \multirow{2}{*}{ 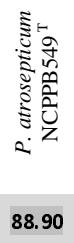 } & \multirow{2}{*}{ 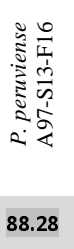 } & \multirow{2}{*}{ 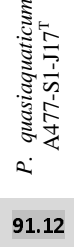 } & \multirow{2}{*}{ 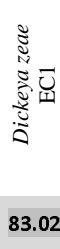 } \\
\hline 1 & $\begin{array}{l}\text { Pectobacterium colocasium } \\
\text { PL152 }^{\mathrm{T}}\end{array}$ & & & & & & & & & & & & & & & & & & & & & \\
\hline 2 & P. aquaticum $\mathrm{A} 212-\mathrm{S} 19-\mathrm{A} 16^{\mathrm{T}}$ & 41.3 & & 89.64 & 91.99 & 88.42 & 89.25 & 93.50 & 93.2 & 94.05 & 90.08 & 94.45 & 88.76 & 93.43 & 88.73 & 88.91 & 93.82 & 88.89 & 89.53 & 88.75 & 95.62 & 82.87 \\
\hline 3 & P. polonicum $\mathrm{DPMP} 315^{\mathrm{T}}$ & 36.4 & 38.8 & & 89.34 & 87.71 & 89.16 & 89.6 & 89.44 & 90.26 & 88.84 & 90.13 & 90.98 & 89.89 & 91.07 & 93.96 & 90.41 & 88.92 & 89.53 & 88.78 & 89.63 & 82.99 \\
\hline 4 & P. actinidae $\mathrm{KKH}^{\mathrm{T}}$ & 39.2 & 46.3 & 37.7 & & 82.90 & 88.94 & 91.79 & 91.48 & 93.25 & 89.71 & 93.02 & 88.53 & 93 & 88.47 & 88.54 & 92.09 & 88.57 & 89.22 & 88.31 & 91.12 & 82.90 \\
\hline 5 & P. fontis M0222 ${ }^{\mathrm{T}}$ & 34.8 & 35.2 & 33.6 & 35.4 & & 87.59 & 88.40 & 88.31 & 88.63 & 88.22 & 88.50 & 87.36 & 88.42 & 87.33 & 87.37 & 89.05 & 87.37 & 87.85 & 86.97 & 88.69 & 82.89 \\
\hline 6 & P. betavasculorum $\mathrm{NCPPB} 2795^{\mathrm{T}}$ & 36.6 & 37.7 & 37.5 & 36.8 & 33.4 & & 89.51 & 89.53 & 89.47 & 89.01 & 89.44 & 89.47 & 89.25 & 89.3 & 89.15 & 90.15 & 91.35 & 92.15 & 91.25 & 89.57 & 82.98 \\
\hline 7 & P. polaris $\mathrm{NIBIO}^{2} 006^{\mathrm{T}}$ & 41.8 & 53.3 & 38.3 & 45.2 & 35.2 & 38.3 & & 93.97 & 93.47 & 90.36 & 93.81 & 88.98 & 92.77 & 88.92 & 89.06 & 96.58 & 89.04 & 89.73 & 88.84 & 93.47 & 82.93 \\
\hline 8 & P. brasiliense LMG21371 ${ }^{\mathrm{T}}$ & 42.5 & 51.7 & 37.5 & 43.8 & 34.9 & 38.1 & 54.4 & & 93.23 & 90.64 & 92.90 & 88.88 & 92.36 & 88.83 & 89.14 & 94 & 89 & 89.59 & 88.78 & 93.94 & 83.05 \\
\hline 9 & P. carotovorum $\mathrm{NCPPB}^{1} 12^{\mathrm{T}}$ & 41.4 & 55.6 & 40.2 & 51 & 35.7 & 38.1 & 52.4 & 51 & & 90.29 & 95.60 & 89.02 & 95.19 & 88.94 & 89.08 & 93.81 & 89.10 & 89.81 & 89.05 & 92.99 & 83 \\
\hline 10 & $P$. aroidearum $\mathrm{L} 6$ & 54.2 & 40.1 & 36.3 & 38.8 & 34.6 & 37.1 & 40.4 & 41.3 & 40.2 & & 90.05 & 88.59 & 90.00 & 88.53 & 88.81 & 91 & 88.97 & 88.98 & 88.21 & 90.35 & 82.97 \\
\hline 11 & P. versatile $\mathrm{CFBP} 6051^{\mathrm{T}}$ & 40.8 & 57.8 & 39.6 & 50 & 35.4 & 38 & 53.5 & 49.2 & 64.2 & 39.5 & & 88.92 & 95.01 & 88.86 & 88.98 & 94.33 & 89.06 & 89.71 & 89.08 & 93 & 82.87 \\
\hline 12 & P. wasabiae CFBP $3304^{\mathrm{T}}$ & 35.6 & 36.6 & 42.8 & 35.8 & 32.9 & 38.5 & 36.8 & 36.3 & 36.8 & 36 & 36.5 & & 88.88 & 93.98 & 91.17 & 89.72 & 89.13 & 90.09 & 89.14 & 88.67 & 83.05 \\
\hline 13 & P. odoriferum BC S7 & 40.4 & 52.6 & 39.2 & 50.4 & 35.6 & 37.8 & 49.2 & 47.4 & 61.4 & 39.5 & 59.8 & 36.7 & & 88.79 & 88.81 & 93.15 & 88.82 & 89.6 & 88.94 & 92.56 & 82.93 \\
\hline 14 & P. parmentieri RNS08-42-1A ${ }^{\mathrm{T}}$ & 35.4 & 36.4 & 43.1 & 35.6 & 32.6 & 37.8 & 36.6 & 36.2 & 36.7 & 35.7 & 36.4 & 54.7 & 36.5 & & 91.40 & 89.74 & 89.01 & 89.88 & 88.85 & 88.97 & 82.98 \\
\hline 15 & P. punjabense $\mathrm{SS} 95^{\mathrm{T}}$ & 36 & 36.9 & 54.6 & 35.5 & 32.8 & 37.3 & 36.9 & 36.8 & 36.8 & 36.3 & 36.4 & 43.3 & 36.3 & 44.1 & & 89.58 & 88.82 & 89.55 & 88.81 & 89.26 & 83.10 \\
\hline 16 & P. parvum $\mathrm{Y} 1$ & 44.7 & 55.1 & 41.3 & 47.1 & 37.2 & 40.3 & 71.3 & 55.2 & 54 & 43 & 56.7 & 39.5 & 51.5 & 39.1 & 39.2 & & 89.69 & 90.44 & $* 73.9$ & 93.85 & 82.62 \\
\hline 17 & P. zantedeschiae $\mathrm{PC} 2$ & 35.3 & 36.1 & 36.3 & 35.4 & 32.9 & 43.8 & 36.5 & 36.3 & 36.4 & 36.5 & 36.2 & 37.2 & 35.7 & 36.7 & 36.1 & 38.6 & & 91.18 & 90.83 & 88.43 & 82.73 \\
\hline 18 & P. atrosepticum $\mathrm{NCPPB}_{549} \mathrm{~T}^{\mathrm{T}}$ & 36.4 & 38.2 & 38.2 & 37.4 & 33.8 & 46.8 & 38.5 & 38 & 38.8 & 36.6 & 38.3 & 40.0 & 38.4 & 39.4 & 38.4 & 41.1 & 43.5 & & 92.96 & 89.49 & 83 \\
\hline 19 & P. peruviense A97-S13-F16 & 34.6 & 35.9 & 35.8 & 34.9 & 31.6 & 43.6 & 36.2 & 35.7 & 36.5 & 34.3 & 36.1 & 36.8 & 36.2 & 36 & 35.5 & 60.1 & 42 & 50.3 & & $* 68.7$ & 83.18 \\
\hline 20 & P. quasiaquaticum $\mathrm{A} 477-\mathrm{S} 1-\mathrm{J} 17^{\mathrm{T}}$ & 43.1 & 64.2 & 39.1 & 42.8 & 36.1 & 38.3 & 53.5 & 55.1 & 50.8 & 41.2 & 50.8 & 36.7 & 48.3 & 36.5 & 37.8 & 55.5 & 35.5 & 38.7 & 50.4 & & 82.25 \\
\hline 21 & Dickeya zeae EC1 & 21.2 & 20.8 & 20.8 & 21.2 & 21.1 & 20.8 & 20.9 & 21.1 & 20.9 & 21.2 & 20.7 & 21.2 & 21.2 & 20.8 & 20.6 & 20.4 & 20.5 & 20.8 & 20.3 & & \\
\hline & & & & & & & & & IDDF & & & & & & & & & & & & & \\
\hline
\end{tabular}

*ANI calculated using EzBiocloud software ANI calculator incorporating OrthoANIu algorithm. 


\section{PHENOTYPIC CHARACTERIZATION}

Biochemical analysis of novel strain PL152 ${ }^{\mathrm{T}}$ was performed with Biolog GEN III MicroPlate

(Biolog, Inc., Hayward, CA), utilizing 71 carbon source utilization and 23 chemical

sensitivity assays. The pure bacterial culture was streaked on BUG agar (Biolog, Inc.) and

incubated for 24 hours at $\pm 28^{\circ} \mathrm{C}$. Colonies were picked using the sterile loops and inoculated into inoculating fluid B to prepare the inoculum. Inoculum (100 ul) was dispensed into each well of a 96-well GEN III microplate. Plates were incubated for 24 hours at $28^{\circ} \mathrm{C}$ and read using the BIOLOG microplate reader; $P$. colocasium utilized $\alpha$-D-Glucose, Pectin, $\alpha$-DLactose, D-Mannose, D-Mannitol, D-Galacturonic Acid, Methyl Pyruvate, D-Fructose, LGalactonic Acid Lactone, $\beta$-Methyl-D-Glucoside, D-Galactose, myo-Inositol, D-Gluconic Acid, D-Cellobiose, D-Salicin, Glycerol, L-Aspartic Acid, Citric Acid, Gentiobiose, NAcetyl-D-Glucosamine, D-Glucose-6-PO4, Sucrose, D-Fructose-6-PO4, Mucic Acid, LRhamnose, D-Aspartic Acid, L-Malic Acid, Acetic Acid, D-Serine, L-Serine, D-Saccharic Acid, Bromo-Succinic Acid; and weak utilization of Dextrin, D-Melibiose, D-Trehalose, LGlutamic Acid, Glucuronamide, Acetoacetic Acid and Formic Acid (Table 2). In this test, PL152 ${ }^{\mathrm{T}}$ was not able to utilize D-Raffinose, D-Sorbitol, Gelatin, p-Hydroxy-Phenylacetic Acid, Tween 40, Glycyl-L-Proline, $\gamma$-Amino-Butyric Acid, D-Maltose, D-Arabitol, LAlanine, D-Lactic Acid Methyl Ester, $\alpha$-Hydroxy-Butyric Acid, L-Arginine, L-Lactic Acid, $\beta$-Hydroxy-D,L-Butyric Acid, 3-Methyl Glucose, D-Glucuronic Acid, $\alpha$-Keto-Butyric Acid, D-Fucose, $\alpha$-Keto-Glutaric Acid, N-Acetyl- $\beta$-D-Mannosamine, L-Fucose, L-Histidine, DMalic Acid, Propionic Acid, D-Turanose, N-Acetyl-D-Galactosamine, L-Pyroglutamic Acid, Quinic Acid, Stachyose, N-AcetylNeuraminic Acid and Inosine (Table 2). In addition, PL152 ${ }^{\mathrm{T}}$ was sensitive to $1 \% \mathrm{NaCl}, 1 \%$ Sodium Lactate, Troleandomycin, Vancomycin, $\mathrm{pH} 6$, 4\% NaCl, Rifamycin SV, Guanidine $\mathrm{HCl}$, Tetrazolium Violet, Lithium Chloride, Niaproof 4 and Tetrazolium Blue; while insensitive to Nalidixic Acid, Aztreonam, pH 5, Minocycline, 
Potassium Tellurite and Sodium Bromate, as per results of chemical sensitivity assay (Table 2).

The proposed novel strain PL152 ${ }^{\mathrm{T}}$ was further tested for antibiotic sensitivity using the disc diffusion method. The bacterial inoculum of $100 \mu 1$ (0.5 OD value) of novel strain PL152 ${ }^{\mathrm{T}}$ was uniformly spread onto Nutrient Agar medium supplemented with $0.4 \%$ dextrose. A commercial disc was impregnated with $20 \mu \mathrm{l}$ of Penicillin $(50 \mathrm{mg} / \mathrm{ml})$, Kanamycin (50 $\mathrm{mg} / \mathrm{ml})$, Tetracycline (40 mg/ml), Chloramphenicol (50 mg/ml), Carbenicillin (100 mg/ml), Gentamicin $(50 \mathrm{mg} / \mathrm{ml})$ and Bacitracin $(50 \mathrm{mg} / \mathrm{ml})$ and placed at the center of the plates containing inoculated media.. After an incubation period of 24 hours at $\pm 28^{\circ} \mathrm{C}$, a "zone of inhibition" was observed determining the sensitivity of the novel strain $P L 152^{\mathrm{T}}$ to the antibiotics (Figure 4).

Table 2. Phenotypic characterization of Pectobacterium colocasium $\mathrm{PL} 152^{\mathrm{T}}{ }^{(=\mathrm{ICMP}} 24362^{\mathrm{T}}$; LMG $32536^{\mathrm{T}}$ ) using Biolog GEN III MicroPlate analyzing 71 carbon source utilization assays and 23 chemical sensitivity assays. Microplate incubated at $28^{\circ} \mathrm{C}$ was read using Biolog's Microbial Identification System software after 24 hours.

\begin{tabular}{llll}
\hline $\begin{array}{l}\text { Characteristics (Carbon } \\
\text { Source/Chemical Sensitivity) }\end{array}$ & PL152 $^{\text {T }}$ & $\begin{array}{l}\text { Characteristics (Carbon } \\
\text { Source/Chemical Sensitivity) }\end{array}$ & PL152 $^{\text {T }}$ \\
\hline D-Raffinose & - & Sucrose & + \\
$\alpha$-D-Glucose & + & N-Acetyl- $\beta$-D-Mannosamine & - \\
D-Sorbitol & - & L-Fucose & - \\
Gelatin & - & D-Fructose-6-PO4 & + \\
Pectin & + & L-Histidine & - \\
p-Hydroxy-Phenylacetic Acid & - & Mucic Acid & + \\
Tween 40 & - & D-Malic Acid & - \\
Dextrin & $++/-)$ & Propionic Acid & - \\
$\alpha$-D-Lactose & + & D-Turanose & - \\
D-Mannose & + & N-Acetyl-D-Galactosamine & - \\
D-Mannitol & + & L-Rhamnose & + \\
Glycyl-L-Proline & - & D-Aspartic Acid & + \\
D-Galacturonic Acid & + & L-Pyroglutamic Acid & - \\
Methyl Pyruvate & + & Quinic Acid & - \\
$\gamma$-Amino-Butyric Acid & - & L-Malic Acid & + \\
D-Maltose & - & Acetic Acid & + \\
D-Melibiose & $+/-)$ & Stachyose & - \\
D-Fructose & + & N-Acetyl Neuraminic Acid & -
\end{tabular}




\begin{tabular}{llll} 
D-Arabitol & - & Inosine & - \\
L-Alanine & - & D-Serine & + \\
L-Galactonic Acid Lactone & + & L-Serine & + \\
D-Lactic Acid Methyl Ester & - & D-Saccharic Acid & + \\
$\alpha$-Hydroxy-Butyric Acid & - & Bromo-Succinic Acid & + \\
D-Trehalose & $(+/-)$ & Formic Acid & $(+/-)$ \\
$\beta$-Methyl-D-Glucoside & + & $1 \%$ NaCl & + \\
D-Galactose & + & $1 \%$ Sodium Lactate & + \\
myo-Inositol & + & Troleandomycin & + \\
L-Arginine & - & Linomycin & $(+/-)$ \\
D-Gluconic Acid & + & Vancomycin & + \\
L-Lactic Acid & - & Nalidixic Acid & - \\
$\beta$-Hydroxy-D,L-Butyric Acid & - & Aztreonam & - \\
D-Cellobiose & + & pH 6 & + \\
D-Salicin & + & $4 \%$ NaCl & + \\
3-Methyl Glucose & - & Fusidic Acid & ++ - \\
Glycerol & + & Rifamycin SV & + \\
L-Aspartic Acid & + & Guanidine HCl & + \\
D-Glucuronic Acid & - & Tetrazolium Violet & + \\
Citric Acid & + & Lithium Chloride & + \\
$\alpha$-Keto-Butyric Acid & - & Sodium Butyrate & $(+/-)$ \\
Gentiobiose & + & pH 5 & - \\
N-Acetyl-D-Glucosamine & + & $8 \%$ NaCl & $(+/-)$ \\
D-Fucose & - & D-Serine & $(+/-)$ \\
D-Glucose-6-PO4 & + & Minocycline & - \\
L-Glutamic Acid & $(+/-)$ & Niaproof 4 & + \\
Glucuronamide & $(+/-)$ & Tetrazolium Blue & + \\
$\alpha$-Keto-Glutaric Acid & - & Potassium Tellurite & - \\
Acetoacetic Acid & $(+/-)$ & Sodium Bromate & - \\
\hline & & & + \\
\hline
\end{tabular}

\section{DESCRIPTION OF PECTOBACTERIUM COLOCASIUM SP. NOV.}

Pectobacterium colocasium (co.lo.ca'si.um L. neut. gen. n. colocasiae, pertaining to taro from which strains PL152 ${ }^{\mathrm{T}}$ and PL155 were isolated).

Gram negative pectinolytic bacteria forms cavities on CVP medium and grows optimally within 24 hours on Nutrient Agar supplemented with $0.4 \%$ dextrose at $28^{\circ} \mathrm{C}$. The colonies were smooth, moist consistency and ceramic white on solid SOB media ( $2 \%$ tryptone, $0.5 \%$ yeast extract, $10 \mathrm{mM} \mathrm{NaCl}, 2.5 \mathrm{mM} \mathrm{KCl}, 10 \mathrm{mM} \mathrm{MgSO}_{4}, 1.5 \%$ agar supplemented with $2 \%$ glycerol) after 2 days at $28^{\circ} \mathrm{C}$. Sensitive to antibiotics: Kanamycin, Tetracycline, Cephalosporin, Carbenicillin, Gentamicin and Bacitracin. Able to utilize $\alpha$ D-Glucose, Pectin, a D-Lactose, D-Mannose, D-Mannitol, D-Galacturonic Acid, Methyl Pyruvate, D-Fructose, L-Galactonic Acid Lactone, $\beta$-Methyl-D-Glucoside, D-Galactose, myo-Inositol, D-Gluconic 
Acid, D-Cellobiose, D-Salicin, Glycerol, L-Aspartic Acid, Citric Acid, Gentiobiose, NAcetyl-D-Glucosamine, D-Glucose-6-PO4, Sucrose, D-Fructose-6-PO4, Mucic Acid, LRhamnose, D-Aspartic Acid, L-Malic Acid, Acetic Acid, D-Serine, L-Serine, D-Saccharic Acid and Bromo-Succinic Acid. Sensitive to $1 \% \mathrm{NaCl}, 1 \%$ Sodium Lactate, Troleandomycin, Vancomycin, pH 6, 4\% NaCl, Rifamycin SV, Guanidine HCl, Tetrazolium Violet, Lithium Chloride, Niaproof 4 and Tetrazolium Blue.

The type strain PL152 ${ }^{\mathrm{T}}$ (ICMP 24362 ${ }^{\mathrm{T}}$; LMG $32536^{\mathrm{T}}$ ) and clonal strain PL155 were isolated in 2018 from infected taro corms in Hawai'i. The DNA G+C content of type strain is 54.6 $\operatorname{mol} \%$.

\section{AUTHOR STATEMENTS}

\section{Conflicts of interest}

The author(s) declare that there are no conflicts of interest.

\section{Funding information}

This work was supported by the USDA National Institute of Food and Agriculture, Hatch project $9038 \mathrm{H}$, managed by the College of Tropical Agriculture and Human Resources. Research was also supported by NIGMS of the National Institutes of Health under award number P20GM125508. The strains were maintained by the funding support from National Science Foundation (NSF-CSBR Grant No. DBI-1561663).

\section{Ethical approval}


Consent for publication

N/A

\section{REFERENCES}

1. Hugouvieux-Cotte-Pattat N, Condemine G, Shevchik VE. Bacterial pectate lyases, structural and functional diversity. Environ Microbiol Rep 2014;6:427-440.

2. Arizala D, Arif M. Genome-wide analyses revealed remarkable heterogeneity in pathogenicity determinants, antimicrobial compounds, and CRISPR-cas systems of complex phytopathogenic genus Pectobacterium. Pathogens 2019;8:247.

3. Mansfield J, Genin S, Magori S, Citovsky V, Sriariyanum M, et al. Top 10 plant pathogenic bacteria in molecular plant pathology. Mol Plant Pathol 2012;13:614-629.

4. Czajkowski R, Perombelon MC, van Veen JA, van der Wolf JM. Control of blackleg and tuber soft rot of potato caused by Pectobacterium and Dickeya species: a review. Plant Pathol 2011;60:999-1013.

5. Glasner JD, Marquez-Villavicencio M, Kim H-S, Jahn CE, Ma B, et al. Nichespecificity and the variable fraction of the Pectobacterium pan-genome. Mol Plant Microbe Interact 2008;21:1549-1560.

6. Zhang Y, Fan Q, Loria R. A re-evaluation of the taxonomy of phytopathogenic genera Dickeya and Pectobacterium using whole-genome sequencing data. Syst Appl Microbiol 2016;39:252-259.

7. Arif M, Czajkowski R, Chapman TA. Editorial: Genome-wide analyses of Pectobacterium and Dickeya species. Front Plant Sci 2022; doi:10.3389/fpls.2022.855262. 
8. Portier P, Pédron J, Taghouti G, Fischer-Le Saux M, Caullireau E, et al. Elevation of Pectobacterium carotovorum subsp. odoriferum to species level as Pectobacterium odoriferum sp. nov., proposal of Pectobacterium brasiliense sp. nov. and Pectobacterium actinidiae sp. nov., emended description of Pectobacterium carotovorum and description of Pectobacterium versatile sp. nov., isolated from streams and symptoms on diverse plants. Int J Syst Evol Microbiol 2019;69:3207-3216.

9. Koh YJ, Kim GH, Lee YS, Sohn SH, Koh HS, et al. Pectobacterium carotovorum subsp. actinidiae subsp. nov., a new bacterial pathogen causing canker-like symptoms in yellow kiwifruit, Actinidia chinensis. N Z J Crop Hortic Sci 2012;40:269-279.

10. Shirshikov FV, Korzhenkov AA, Miroshnikov KK, Kabanova AP, Barannik AP, et al. Draft genome sequences of new genomospecies "Candidatus Pectobacterium maceratum" strains, which cause soft rot in plants. Genome Announc 2018;6:e00260-18.

11. Duarte V, De Boer SH, Ward L de, De Oliveira AMR. Characterization of atypical Erwinia carotovora strains causing blackleg of potato in Brazil. J Appl Microbiol 2004;96:535-545.

12. Nabhan S, De Boer SH, Maiss E, Wydra K. Taxonomic relatedness between Pectobacterium carotovorum subsp. carotovorum, Pectobacterium carotovorum subsp. odoriferum and Pectobacterium carotovorum subsp. brasiliense subsp. nov. J Appl Microbiol 2012;113:904-913.

13. Hauben L, Moore ER, Vauterin L, Steenackers M, Mergaert J, et al. Phylogenetic position of phytopathogens within the Enterobacteriaceae. Syst Appl Microbiol 1998;21:384-397.

14. Pedron J, Bertrand C, Taghouti G, Portier P, Barny M-A. Pectobacterium aquaticum 
sp. nov., isolated from waterways. Int J Syst Evol Microbiol 2019;69:745-751.

15. Nabhan S, De Boer SH, Maiss E, Wydra K. Pectobacteriumaroidearum sp. nov., a soft rot pathogen with preference for monocotyledonous plants. Int J Syst Evol Microbiol 2013;63:2520-2525.

16. Gardan L, Gouy C, Christen R, Samson R. Elevation of three subspecies of Pectobacterium carotovorum to species level: Pectobacterium atrosepticum sp. nov., Pectobacterium betavasculorum sp. nov. and Pectobacterium wasabiae sp. nov. Int J Syst Evol Microbiol 2003;53:381-391.

17. Oulghazi S, Cigna J, Lau YY, Moumni M, Chan KG, et al. Transfer of the waterfall source isolate Pectobacterium carotovorum M022 to Pectobacterium fontis sp. nov., a deep-branching species within the genus Pectobacterium. Int J Syst Evol Microbiol 2019;69:470-475.

18. Sarfraz S, Riaz K, Oulghazi S, Cigna J, Sahi ST, et al. Pectobacterium punjabense sp. nov., isolated from blackleg symptoms of potato plants in Pakistan. Int J Syst Evol Microbiol 2018;68:3551-3556.

19. Foss S, Harder J. In Validation of the publication of new names and new combinations previously effectively published outside the IJSB. List No. 68. Int J Syst Bacteriol 1999;49:1-3.

20. Alcorn SM, Orum TV, Steigerwalt AG, Foster JL, Fogleman JC, et al. Taxonomy and pathogenicity of Erwinia cacticida sp. nov. Int J Syst Evol Microbiol 1991;41:197212.

21. Portier P, Pédron J, Taghouti G, Dutrieux C, Barny M-A. Updated taxonomy of 
Pectobacterium genus in the CIRM-CFBP bacterial collection: when newly described species reveal “old” endemic population. Microorganisms 2020;8:1441.

22. Khayi S, Cigna J, Chong TM, Quêtu-Laurent A, Chan K-G, et al. Transfer of the potato plant isolates of Pectobacterium wasabiae to Pectobacterium parmentieri sp. nov. Int J Syst Evol Microbiol 2016;66:5379-5383.

23. Pasanen M, Waleron M, Schott T, Cleenwerck I, Misztak A, et al. Pectobacterium parvum sp. nov., having a Salmonella SPI-1-like Type III secretion system and low virulence. Int J Syst Evol Microbiol 2020;70:2440.

24. Dees MW, Lysøe E, Rossmann S, Perminow J, Brurberg MB. Pectobacterium polaris sp. nov., isolated from potato (Solanum tuberosum). Int J Syst Evol Microbiol 2017;67:5222-5229.

25. Waleron M, Misztak A, Waleron M, Jonca J, Furmaniak M, et al. Pectobacterium polonicum sp. nov. isolated from vegetable fields. Int J Syst Evol Microbiol 2019;69:1751-1759.

26. Moussa HB, Pédron J, Bertrand C, Hecquet A, Barny M-A. Pectobacterium quasiaquaticum sp. nov., isolated from waterways. Int J Syst Evol Microbiol 2021;71:005042.

27. Waleron M, Misztak A, Waleron M, Franczuk M, Jońca J, et al. Pectobacterium zantedeschiae sp. nov. a new species of a soft rot pathogen isolated from Calla lily (Zantedeschia spp.). Syst Appl Microbiol 2019;42:275-283.

28. Waleron M, Misztak A, Waleron M, Franczuk M, Wielgomas B, et al. Transfer of Pectobacterium carotovorum subsp. carotovorum strains isolated from potatoes grown at 
high altitudes to Pectobacterium peruviense sp. nov. Syst Appl Microbiol 2018;41:85-93.

29. Wayne LG, Brenner DJ, Colwell RR, Grimont PAD, Kandler O, et al. Report of the ad hoc committee on reconciliation of approaches to bacterial systematics. Int J Syst Evol Microbiol 1987;37:463-464.

30. Hélias V, Hamon P, Huchet E, Wolf JVD, Andrivon D. Two new effective semiselective crystal violet pectate media for isolation of Pectobacterium and Dickeya. Plant Pathol 2012;61:339-345.

31. Arizala D, Dobhal S, Babler B, Crockford AB, Rioux RA, et al. Development of a multiplex TaqMan qPCR targeting unique genomic regions for the specific and sensitive detection of Pectobacterium species and P. parmentieri. J Appl Microbiol 2022; doi.org/10.1111/jam.15447

32. Dobhal S, Boluk G, Babler B, Stulberg MJ, Rascoe J, et al. Comparative genomics reveals signature regions used to develop a robust and sensitive multiplex TaqMan realtime qPCR assay to detect the genus Dickeya and Dickeya dianthicola. J Appl Microbiol 2020;128:1703-1719.

33. Boluk G, Arizala D, Ocenar J, Mokwele J, Silva J, et al. First report of Pectobacterium brasiliense causing soft rot on Brassica oleracea var. sabellica in Hawaii, United States. Plant Dis 2020;104:2721.

34. Kearse M, Moir R, Wilson A, Stones-Havas S, Cheung M, et al. Geneious Basic: an integrated and extendable desktop software platform for the organization and analysis of sequence data. Bioinformatics 2012;28:1647-1649.

35. Tamura K, Stecher G, Kumar S. MEGA11: molecular evolutionary genetics analysis 
version 11. Mol Biol Evol 2021;38:3022-3027.

36. De Vos P. Multilocus sequence determination and analysis. In: Methods in microbiology. Elsevier; 2011. pp. 385-407.

37. Wick RR, Judd LM, Gorrie CL, Holt KE. Unicycler: resolving bacterial genome assemblies from short and long sequencing reads. PLoS Comput Biol 2017;13:e1005595.

38. Davis JJ, Wattam AR, Aziz RK, Brettin T, Butler R, et al. The PATRIC Bioinformatics Resource Center: expanding data and analysis capabilities. Nucleic Acids Res 2020;48:D606-D612.

39. Brettin T, Davis JJ, Disz T, Edwards RA, Gerdes S, et al. RASTtk: a modular and extensible implementation of the RAST algorithm for building custom annotation pipelines and annotating batches of genomes. Sci Rep 2015;5:1-6.

40. Yoon S-H, Ha S-M, Lim J, Kwon S, Chun J. A large-scale evaluation of algorithms to calculate average nucleotide identity. Antonie Van Leeuwenhoek 2017;110:1281-1286.

41. Meier-Kolthoff JP, Carbasse JS, Peinado-Olarte RL, Göker M. TYGS and LPSN: a database tandem for fast and reliable genome-based classification and nomenclature of prokaryotes. Nucleic Acids Res 2022;50:D801-D807.

42. Konstantinidis KT, Tiedje JM. Genomic insights that advance the species definition for prokaryotes. Proc Natl Acad Sci 2005;102:2567-2572. 


\section{FIGURES AND TABLES}

Figure 1. Timeline of major taxonomic events based on taxonomic description of Pectobacterium.

Figure 2. Multi-Locus Sequence Analysis (MLSA) of 54 strains, comprising Pectobacterium colocasium (PL152 ${ }^{\mathrm{T}}$; ICMP 24362 ${ }^{\mathrm{T}}$; LMG $32536^{\mathrm{T}}$ ) (brown color) and other known Pectobacterium sp. and Dickeya zeae EC1 (as an outgroup), was perfomred using nine housekeeping genes (dnaA, gapA, gyrB, recA, dnaN, rpoS, mdh, rpoA and $d n a K)$. MEGA11 (Maximum Likelihood method) with a bootstrap test of 1,000 replicates was used for analysis. Bootstrap values are indicated at nodes. Pectobacterium colocasium formed a novel clade.

Figure 3. The $16 \mathrm{~S}$ rRNA tree was generated using MEGA 11(Maximum Likelihood method) for all type strains within Pectobacterium: P. actinidiae $\mathrm{KKH}^{\mathrm{T}}, P$. carotovorum NCPPB $312^{\mathrm{T}}$, P. aroidearum SCRI109 ${ }^{\mathrm{T}}$, P. brasiliense $\mathrm{LMG} 1371^{\mathrm{T}}$, P. polaris $\mathrm{NIBIO} 1006^{\mathrm{T}}$, P. parvum $\mathrm{s} 0421^{\mathrm{T}}$, P. odoriferum $\mathrm{LMG} 7566^{\mathrm{T}}$, P. versatile $\mathrm{CFBP} 6051^{\mathrm{T}}$, P. aquaticum A212S19-A $16^{\mathrm{T}}$, P. zantedeschiae $9 \mathrm{M}^{\mathrm{T}}$, P. fontis $\mathrm{M} 022^{\mathrm{T}}$, P. polonicum $\mathrm{DPMP} 315^{\mathrm{T}}$, P. punjabense $\mathrm{SS}^{\mathrm{T}}{ }^{\mathrm{T}}, \quad$ P. parmentieri $\mathrm{RNS} 08-42-1 \mathrm{~A}^{\mathrm{T}}, \quad$ P. wasabiae $\mathrm{CFBP}_{304}{ }^{\mathrm{T}}, \quad$ P. atrosepticum ${\text { LMG } 2386^{\mathrm{T}}, P \text {. peruviense IFB5232 }}^{\mathrm{T}}$, P. betavasculorum CFBP2122 ${ }^{\mathrm{T}}$, P. colocasium PL152 ${ }^{\mathrm{T}}$ and Dickeya zeae $\mathrm{EC}^{\mathrm{T}}$ (as an outgroup). Pectobacterium colocasium strain PL152 ${ }^{\mathrm{T}}$ (ICMP $24362^{\mathrm{T}}$; LMG $32536^{\mathrm{T}}$ ) labelled with red solid circle, rooted among Pectobacterium species.

Figure 4. Antibiotic assay determining the sensitivity of the novel strain PL152 ${ }^{\mathrm{T}}$ (ICMP $24362^{\mathrm{T}}$; LMG $32536^{\mathrm{T}}$ ) to seven different antibiotics- Penicillin (50 mg/ml), Kanamycin (50 mg/ml), Tetracycline (40 mg/ml), Chloramphenicol (50 mg/ml), Carbenicillin (100 mg/ml), 
Gentamicin (50 mg/ml) and Bacitracin (50 mg/ml) (A-G) by forming a "zone of inhibition”. Distilled water was used as control (H).

\section{SUPPLEMENTAL MATERIALS}

Supplemental Figure 1. Initial identification and phylogenetic analysis of Pectobacterium colocasium strains (PL152 ${ }^{\mathrm{T}}$ and PL155), 48 different species of Pectobacterium genus and Dickeya zeae $\mathrm{EC}^{\mathrm{T}}{ }^{\mathrm{T}}$ as an outgroup, using MEGA11 (Maximum likelihood method) with 1,000 bootstrap replication. Partial sequences of three housekeeping genes, dnaA, gapA and gyrB, were cancatenated and used to generate phylogenetic tree. PL152 ${ }^{\mathrm{T}}$ and PL155 are labelled with a red solid circle on the phylogenetic tree, depicting two strains as clonal, rooted among other Pectobacterium species in a novel clade.

Supplemental Table 1. List of 53 bacterial strains including 52 known strains of Pectobacterium species and Dickeya zeae EC1 (as an outgroup) retrieved from NCBI GenBank database for multi-locus sequence analysis.

Supplemental Table 2. List of type strains of known Pectobacterium species and Dickeya zeae $\mathrm{EC} 1$ (as an outgroup), retrieved from the NCBI GenBank database and used for the $16 \mathrm{~S}$ rRNA analysis. 


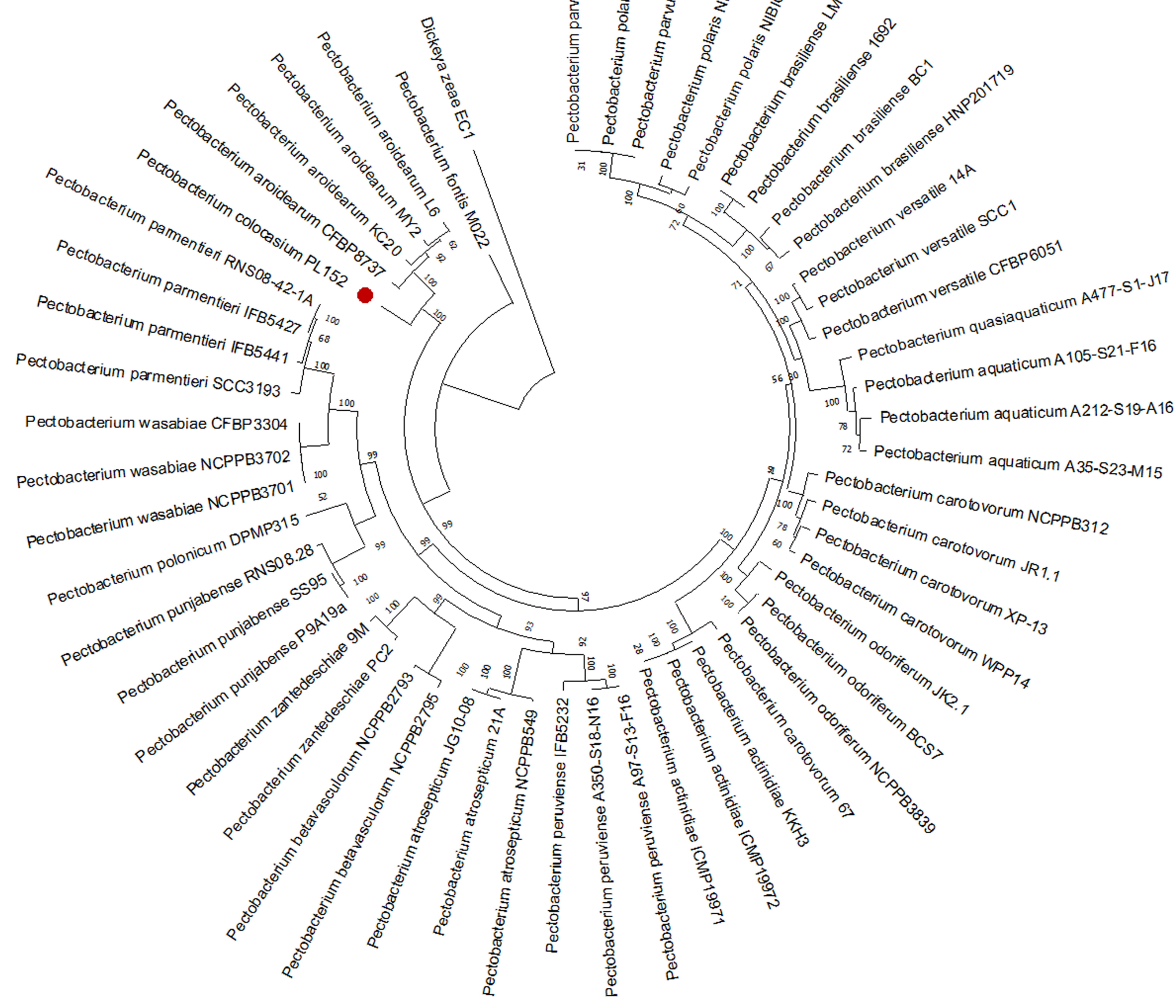




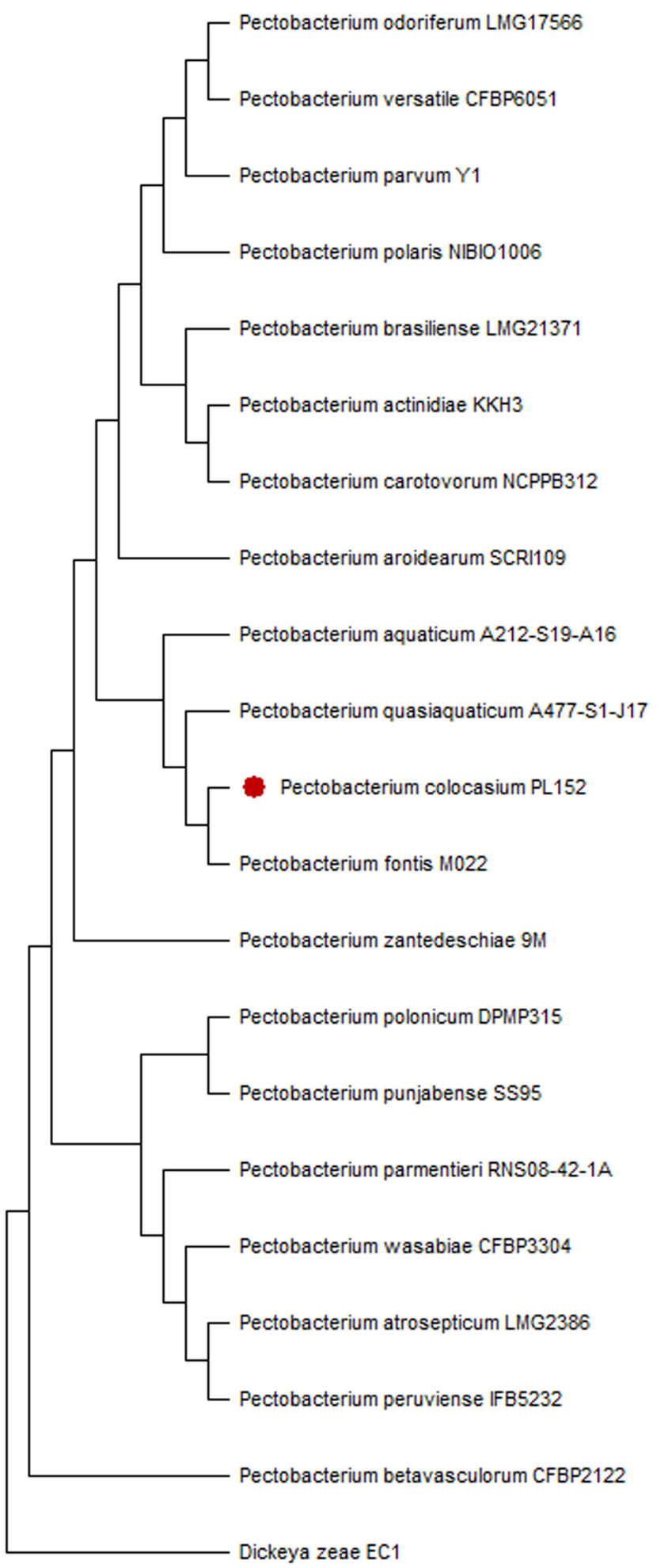


